\title{
A reactive poroelastic model to predict the periprosthetic tissue healing
}

\author{
Dominique Ambard* — Gaëtan Guérin** — Pascal Swider** \\ * Laboratoire de Mécanique et Génie Civil, UMR 5508 \\ Université Montpellier II \\ CC 048 Place Eugène Bataillon \\ F-34095 Montpellier cedex 5 \\ ambard@lmgc.univ-montp2.fr \\ ** Laboratoire de Biomécanique, EA3697 \\ Université Toulouse III \\ CHU Purpan, Place Dr Baylac \\ F-31049 Toulouse cedex \\ \{Guerin,swider\}@cict.fr
}

\begin{abstract}
Conditions influencing the implant osteointegration in the early post-operative period include the surgical technique and coupled mechanical and biochemical factors. We hypothesized that coupling deformable porous media mechanics to governing equations of cell migration, might help to predict the periprosthetic tissue healing and in particular the heterogeneous bone formation which is unfavourable to the implant survival. To proceed, a multiphasic model of porous tissue surrounding a loaded implant was coupled to osteoblast migration and immature bone deposit. A finite element resolution was implemented and the application concerned a canine implant. The sensitivity analysis using volume strain as variable showed that compression was rather unfavourable to homogenous distribution of periprosthetic bone healing.

RÉSUMÉ. L'ostéointégration des implants post-opératoire est influencée par la technique chirurgicale et des facteurs mécaniques et biochimiques. Nous avons supposé qu'une approche poroélastique associée à la notion de milieu réactif et aux équations biomathématiques de migration cellulaire pouvait permettre de prévoir la cicatrisation périprothétique et notamment la formation hétérogène défavorable à la survie de l'implant. Un modèle multiphasique du tissu a été couplé aux équations de migrations ostéoblastiques et à un modèle d'apposition d'os néoformé. Une résolution par la méthode des éléments finis a été développée et appliquée à un modèle expérimental canin. Une étude de sensibilité à la déformation volumique a montré le rôle plutôt défavorable d'une compression sur une cicatrisation homogène.
\end{abstract}

KEYWORDS: biomathematics, osteointegration, poroelastic, finite element, osteoblast.

MOTS-CLÉS : biomathématique, ostéointégration, poroélasticité, éléments finis, ostéoblates.

DOI:10.3166/EJCM.18.131-143 @ 2009 Lavoisier, Paris

EJCM - 18/2009. Numerical models in biomechanics, pages 131 to 143 


\section{Objectifs}

While the quality of implant fixation is determined primarily by the bone and tissue anchoring the implant, conditions influencing bone growth in the early post-operative period include the surgical technique (Hahn et al., 1988) and coupled mechanical (Carter, 1987; Prendergast et al., 1997) and biochemical factors (Bailon-Plaza et al., 2001). Low performances were generally associated with a low mineralization or a strong heterogeneous distribution of bony structure (sclerotic bone rim) in the new-formed surrounding tissue (Søballe et al., 2004; Mouzin et al., 2001; Vestermark et al., 2004).

We hypothesized that coupling deformable porous media mechanics to governing equation of cell migrations, might help to predict the heterogeneity of periprosthetic tissue healing. To proceed, a multiphasic model (solid, fluid, osteoblasts, growth factors) of porous tissue surrounding a stable loaded implant was coupled to osteoblast migration (diffusion, haptotaxis, chemotaxis) and immature bone deposit (reactive media). Application concerned a canine implant(Bechtold et al., 2001a; Bechtold et al., 2001b; Søballe et al., 1993).

The periprosthetic tissue healing is an intramembranous process. Mesenchymal cells differentiate into osteoblastic cells conditionning the bone formation (Alliston et al., 2000; Kibbin, 1997). While delivering growth factors regulating cell proliferation and differentiation and favouring extra-cellular matrix synthesis, the neovascularization is of prime importance in the healing process (Conover, 2000; Linckart et al., 1996). Cells migration mechanisms, such as haptotactic and chemotactic active migrations also showed a significant role (Maheshwari et al., 1998; Puleo et al., 1991; Kunzler et al., 2007; Friedl et al., 1998). The predictive numerical models of cell differentiation processes (Carter, 1987; Prendergast et al., 1997; Van-DerMeulen et al., 2002), bone healing (Carter et al., 1988; Lacroix, 2000; Bailon-Plaza et al., 2001) and bone remodelling (Huiskes et al., 2000) have been developed using structural models or poroelastic approaches. In most of models, the time variable is not explicit so the biological time scale jointed to transient loads is not fully taken into account. These studies provided a significant improvement to bone formation models but the understanding of in vivo mechano-biological phenomena, is still an open problem. In this paper, we proposed an original mechano-biological model associating the governing equation of deformable porous media to biomathematical equations of osteoblastic migration and immature bone deposit considered as a reactive medium. The application concerned a canine implant.

\section{Governing equations}

As shown in Figure 1a, we implemented a multiphasic model that involved four phases at the microscopic scale: the interconnected porous skeleton $\left(\phi_{s}\right)$, the saturat- 
ing fluid phase $\left(\phi_{f}\right)$ for the bone marrow, the osteoblast phase $\left(C_{c}\right)$ and the growth factor phase $\left(C_{M}\right)$. To summarize, the mechanical load induces the intersticial fluid flow that modified the distribution of growth factors and osteoblast phase to form new bone. Cell active migration, related to the growth factor concentration and to the porosity gradient, is also concerned. The mechanical model is established in the quasistatic domain with a negligible mass increase, an incompressible fluid and a field of small strain $(J=1+\operatorname{trace} \varepsilon)$. The balance momentum and the Cauchy tensor $\sigma$ are respectively described by Equations [1] and [2], $p$ being the average pressure in the porous medium.

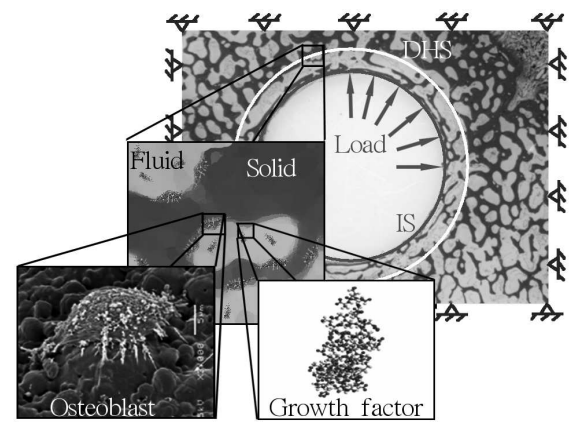

a)

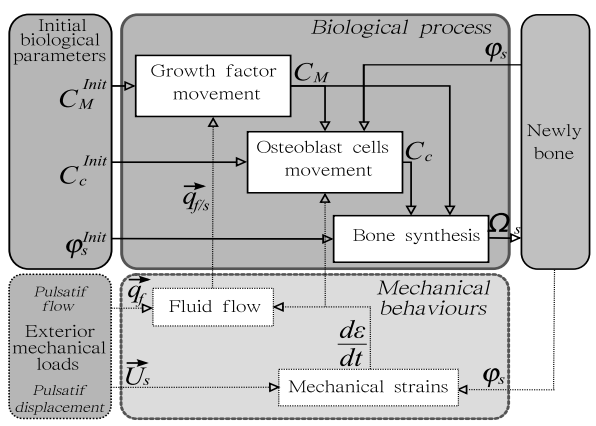

b)

Figure 1. a) Multiphasic model of periprosthetic healing tissue superposed to histomorphometric cut. On this cut, the implant surface $(I S)$ and the drill hole surface $(D H S)$ are showed. b) Diagram of the various couplings between the biological parameters and mechanical stimulus

$$
\begin{aligned}
& \vec{\nabla} \sigma-\vec{\nabla} p=\overrightarrow{0} \\
& \sigma=\frac{v \cdot E}{(1-2 \cdot v) \cdot(1+v)} \cdot \operatorname{trace} \varepsilon \cdot I+\frac{E}{(1+v)} \cdot \varepsilon
\end{aligned}
$$

The Equation [3] of mass conservation is established first assuming that the porous volume element is saturated ( $\phi_{s}+\phi_{f}=1$ ), and second that the volumes of cell phase and growth factor phase are negligible. The fluid flux $\vec{q}_{f / s}$, relative to the porous skeleton, is related to the pressure gradient by the permeability tensor using the Darcy law [4], $\mu$ being the fluid dynamic viscosity and $k$ being the isotropic permeability. Because the biological tissue is considered as a reactive media, material properties are updated using the mineral matrix formation $\left(\phi_{s}\right)$ induced by osteoblasts. It concerns $E, v$ in Equation [2], and the permeability tensor in Equation [4]. A power law is used for the elastic properties (Lacroix, 2000; Fung, 1981) and for the permeability, a Kozeny-Carman model is used inspired by Arramon et al. (2001).

$$
\frac{d J}{d t}=\vec{\nabla} \cdot \vec{q}_{f / s}
$$




$$
\vec{q}_{f / s}=-\frac{k}{\mu} \cdot \vec{\nabla} p
$$

The synthesis of mineral matrix modelled as the source $\Omega_{s}$ in Equation [6], contributes to the mass conservation of the solid fraction expressed by Equation [5]. The synergetic action of osteoblast $C_{c}$ and growth factors $C_{M}$ (Meinel et al., 2003) is promoted by the fluid fraction $\phi_{f}$ and the coefficient of matrix synthesis $\alpha_{s}$.

$$
\begin{aligned}
& \frac{\partial}{\partial t}\left(\phi_{s} . J\right)=\Omega_{s} \\
& \Omega_{s}=\alpha_{s} \cdot \phi_{f}^{2} \cdot C_{c} \cdot C_{M}
\end{aligned}
$$

The growth factors $C_{M}$ are involved into the fluid fraction. Equation [7] expressed the mass conservation as a function of the fluid fraction $\phi_{f}$ (or porosity), the fluid flux $\vec{q}_{f / s}$ and the dispersion tensor $D_{M}$.

$$
\phi_{f} \cdot \frac{\partial C_{M}}{\partial t}=\phi_{f} \cdot D_{M} \cdot \Delta C_{M}+\vec{q}_{f / s} \cdot \vec{\nabla} C_{M}
$$

The conservation of cells is expressed by Equation [8] in which the fluid fraction $\phi_{f}$, the cells flux $\vec{q}_{c / s}$ and the cell source $\Omega_{c}$ interact. This source allows the cell proliferation to be modelled using a logistic law [9], $N_{c}$ and $\alpha_{c}$ respectively being the threshold limit of cells and the proliferation coefficient (Bailon-Plaza et al., 2001). Osteoblasts are lining cells that can migrate using three main processes (Zaman et al., 2005). Firstly, a random diffusion is observed. Cells are moving to the opposite of the concentration gradient $C_{c}$; the diffusive property being $D_{c}$. Second, the chemotactic migration induces a mobility related to the gradient of growth factors $C_{M}$ (Maheshwari et al., 1998). It is combined with $\chi_{c}$, the chemotactic property of the osteoblast phase (Dee-Kay et al., 1999). Third, the haptotactic migration combines the gradient of porous skeleton $\phi_{s}$ to the haptotactic coefficient $h_{c}$. Finally, the cell flux $\vec{q}_{c / s}$ is described by Equation [10].

$$
\begin{aligned}
& \frac{\partial}{\partial t}\left(\phi_{f} \cdot J \cdot C_{c}\right)=\vec{\nabla} \cdot \vec{q}_{c / s}+\Omega_{c} \\
& \Omega_{c}=\alpha_{c} \cdot \phi_{f} \cdot C_{c} \cdot\left(N_{c}-\phi_{f} \cdot C_{c}\right) \\
& \vec{q}_{c / s}=\phi_{f} \cdot\left(D_{c} \cdot \vec{\nabla} C_{c}-\chi_{c} \cdot C_{c} \cdot \vec{\nabla} C_{M}-h_{c} \cdot C_{c} \cdot \vec{\nabla} \phi_{s}\right)
\end{aligned}
$$

In conclusion, the multiphasic model is described by the set of non-linear coupled Equations [1], [3], [5], [7], [8] and the selected variables are the field displacement $U_{s}$ of the porous skeleton, the fluid intersticial pressure $p$, the porous skeleton fraction $\phi_{s}$, the osteoblast concentration $C_{c}$ and the growth factor concentration $C_{M}$.

\section{Finite element formulation}

The set of governing equations is solved using an implicit formulation in time [11] (Ames-William, 1977) and a first order Taylor expansion [12]. We identify the 
capacity term $C\left(x_{j}\right) \cdot \frac{\partial x_{i}}{\partial t}$, the convective term $\overrightarrow{q_{j}}\left(x_{j}\right) \cdot \vec{\nabla} x_{i}$, the diffusive term $\vec{\nabla} \cdot \overrightarrow{q_{i}}\left(x_{j}\right)$ and the source term $\Omega_{i}\left(x_{j}\right)$.

$$
\begin{aligned}
& {\left[C\left(x_{j}\right) \cdot \frac{\partial x_{i}}{\partial t}\right]^{n}+\left[\overrightarrow{q_{j}}\left(x_{j}\right) \cdot \vec{\nabla} x_{i}\right]^{n}=\left[\vec{\nabla} \cdot \overrightarrow{q_{i}}\left(x_{j}\right)\right]^{n}+\left[\Omega_{i}\left(x_{j}\right)\right]^{n}} \\
& f^{n}\left(x_{i}\right) \approx f^{n-1}\left(x_{i}\right)+\left[\frac{\partial f}{\partial x_{i}}\right]^{n-1} \cdot\left(x_{i}^{n}-x_{i}^{n-1}\right)
\end{aligned}
$$

The weak form of Equation [1] is expressed by Equation [13] with $N_{u}$ the matrix of shape function, $B_{\varepsilon}$ the gradient matrix of the displacement filed $U_{s}$ and $B_{I_{1}}$ the matrix of first invariants of strain tensor $\varepsilon$.

$$
\hat{U}_{s}^{n} \cdot \int_{\Im_{e}} B_{\varepsilon}^{T} \cdot K \cdot B_{\varepsilon} \cdot d \Im_{e}+\hat{p}^{n} \cdot \int_{\Im_{e}} B_{I_{1}}^{T} \cdot N_{p} \cdot d \Im_{e}=\int_{\partial \Im_{e}} N_{u}^{T} \cdot \overrightarrow{f_{s}} \cdot d S_{e}
$$

The weak form of Equations [3] and [5] are respectively expressed by Equation [14] and [15] with $B_{p}$ the matrix of gradient functions, $N_{p}$ and $N_{\phi}$ are the matrices of shape functions for $p$ and $\phi_{s}$.

$$
\begin{gathered}
-\hat{p}^{n} \cdot \int_{\Im_{e}} B_{p}^{T} \cdot \frac{k}{\mu} \cdot \Delta t \cdot B_{p} \cdot d \Im_{e}-\left(\hat{U}_{s}^{n}-\hat{U}_{s}^{n-1}\right) \cdot \int_{\Im_{e}} N_{p}^{T} \cdot B_{I_{1}} \cdot d \Im_{e} \\
=-\int_{\partial \Im_{e}} N_{p}^{T} \cdot q_{f / s}{ }^{n} \cdot \Delta t \cdot d S_{e} \\
\left(\hat{\phi}_{s}^{n}-\hat{\phi}_{s}^{n-1}\right) \cdot \int_{\Im_{e}} N_{\phi}^{T} \cdot\left(\frac{1}{\Delta t}-2 \cdot \alpha_{s} \cdot\left(1-\phi_{s}^{n-1}\right)^{2} \cdot C_{M}^{n-1} \cdot C_{c}^{n-1}\right) \cdot N_{\phi} \cdot d \Im_{e} \\
+\left(\hat{U}_{s}^{n}-\hat{U}_{s}^{n-1}\right) \cdot \int_{\Im_{e}} N_{\phi}^{T} \cdot \frac{\phi_{s}^{n-1}}{\Delta t} \cdot B_{I_{1}} \cdot d \Im_{e} \\
+\left(\hat{C}_{m}^{n}-\hat{C}_{M}^{n-1}\right) \cdot \int_{\Im_{e}} N_{\phi}^{T} \cdot \alpha_{s} \cdot\left(1-\phi_{s}^{n-1}\right)^{2} \cdot C_{c}^{n-1} \cdot N_{m} \cdot d \Im_{e} \\
-\left(\hat{C}_{c}^{n}-\hat{C}_{c}^{n-1}\right) \cdot \int_{\Im_{e}} N_{\phi}^{T} \cdot \alpha_{s} \cdot\left(1-\phi_{s}^{n-1}\right)^{2} \cdot C_{M}^{n-1} \cdot N_{c} \cdot d \Im_{e} \\
=\int_{\Im_{e}} N_{\phi}^{T} \cdot \Omega_{s}^{n-1} d \Im_{e}
\end{gathered}
$$

The weak integral form of Equation [7] is given by Equation [16] with $N_{m}$ the matrix of shape function for $C_{M}$ and $B_{m}$ the gradient matrix. The computation of laplacien $\Delta C_{M}$ at integration points is requiered and its computation is achieved according to Dhatt et al. (1984). 


$$
\begin{array}{r}
\hat{C}_{M}^{n} \cdot \int_{\Im_{e}}\left(1-\phi_{s}^{n-1}\right) \cdot\left(B_{m}^{T} \cdot D_{M} \cdot B_{m}+N_{m}^{T} \cdot \frac{1}{\Delta t} \cdot N_{m}\right) \\
+N_{m}^{T} \cdot \frac{k}{\mu} \cdot \vec{\nabla} p^{n-1} \cdot B_{m} \cdot d \Im_{e} \\
+\left(\hat{p}^{n}-\hat{p}^{n-1}\right) \cdot \int_{\Im_{e}} N_{m}^{T} \cdot \frac{k}{\mu} \cdot \vec{\nabla} C_{M}^{n-1} \cdot B_{p} \cdot d \Im_{e} \\
+\left(\hat{\phi}_{s}^{n}-\hat{\phi}_{s}^{n-1}\right) \cdot \int_{\Im_{e}} N_{m}^{T} \cdot\left(\frac{\left(C_{M}^{n-1}-C_{M}^{n-2}\right)}{\Delta t}+D_{M} \cdot \Delta C_{M}^{n-1}\right) N_{\phi} \cdot d \Im_{e} \\
=\int_{\partial \Im_{e}} N_{m}^{T} \cdot q_{M} \vec{s}^{n}+\hat{C}_{M}^{n-1} \cdot \int_{\Im_{e}} N_{m}^{T} \cdot \frac{1}{\Delta t} \cdot N_{m} \cdot d \Im_{e}
\end{array}
$$

Equation [8] is written in the weak integral form [17] with $N_{o}$ the matrix of shape function for $C_{c}$ and $B_{o}$ the gradient matrix. Here also the computation of laplacien $\triangle C_{M}$ is necessary as far as $\triangle \phi_{s}$.

$$
\begin{array}{r}
\hat{C}_{c}^{n} \cdot \int_{\Im_{e}} \phi_{f}^{n-1} \cdot\left(B_{o}^{T} \cdot D_{c} \cdot B_{o}+N_{o}^{T} \cdot\left(\frac{1}{\Delta t}+\chi_{c} \cdot \Delta C_{M}^{n-1}+h_{c} \cdot \Delta \phi_{s}^{n-1}\right.\right. \\
\left.+\alpha_{c} \cdot\left(N_{c}-2 \cdot \phi_{f}^{n-1} \cdot C_{c}^{n-1}\right)\right) \cdot N_{o} \\
\left.+N_{o}^{T} \cdot\left(\chi_{c} \cdot \vec{\nabla} C_{M}^{n-1}+h_{c} \cdot \vec{\nabla} \phi_{s}^{n-1}\right) \cdot B_{o}\right) \cdot d \Im_{e} \\
+\left(\hat{U}_{s}^{n}-\hat{U}_{s}^{n-1}\right) \cdot \int_{\Im_{e}} N_{o}^{T} \cdot \frac{\phi_{f}^{n-1} \cdot C_{c}^{n-1}}{\Delta t} \cdot B_{u} \cdot d \Im_{e} \\
+\left(\hat{C}_{m}^{n}-\hat{C}_{m}^{n-1}\right) \cdot \int_{\Im_{e}} \phi_{f}^{n-1} \cdot \chi_{c} \cdot\left(N_{o}^{T} \cdot \vec{\nabla} C_{c}^{n-1} \cdot B_{m}+N_{o}^{T} \cdot C_{c}^{n-1} \cdot L_{m}\right) \cdot d \Im_{e} \\
-\left(\hat{\phi}_{s}^{n}-\hat{\phi}_{s}^{n-1}\right) \cdot \int_{\Im_{e}} N_{o}^{T} \cdot\left(-\vec{\nabla} C_{c}^{n-1} \cdot\left(\chi_{c} \cdot \vec{\nabla} C_{M}^{n-1}+h_{c} \cdot \vec{\nabla} \phi_{s}^{n-1}\right)\right. \\
-C_{c}^{n-1} \cdot\left(\chi_{c} \cdot \Delta C_{M}^{n-1}+h_{c} \cdot \Delta \phi_{s}^{n-1}\right)+D_{c} \cdot \Delta C_{c}^{n-1}+\alpha_{c} \cdot \phi_{f}^{n-1} \cdot \Delta C_{M}^{n-1} \\
\left.\left.+h_{c} \cdot \phi_{f}^{n-1} \cdot \Delta \phi_{s}^{n-1}+\frac{C_{c}^{n-1}}{\Delta t}\right) \cdot N_{\phi}+N_{o}^{T} \cdot\left(h_{c} \cdot \phi_{f}^{n-1} \cdot \vec{\nabla} C_{c}^{n-1}\right) \cdot B_{\phi}\right) \cdot d \Im_{e} \\
=\hat{C}_{c}^{n-1} \cdot \int_{\Im_{e}} N_{o}^{T} \cdot \frac{\phi_{f}^{n-1}}{\Delta t} \cdot N_{o} \cdot d \Im_{e} \\
+\int_{\partial \Im_{e}} N_{o}^{T} \cdot q_{c / s}{ }^{n} \cdot d S_{e}+\int_{\Im_{e}} N_{o}^{T} \cdot \Omega_{c}^{n} \cdot d \Im_{e}
\end{array}
$$

Finally, the matrix formulation is expressed by the system [18] after integration (Gauss method). Equations [13],[14],[15], [16], [17] respectively correspond to lines 1, 2, 5, 
3 and 4. This matrix form highlights the strong couplings between phases and the solution is obtained using a linear implicit formulation at each time step.

$$
\begin{aligned}
& \left\|\begin{array}{ccccc}
K_{u u} & -K_{u p} & 0 & 0 & 0 \\
-K_{u p}^{T} & -K_{p p} \cdot \Delta t & 0 & 0 & 0 \\
0 & V_{m p} & K_{m m} & 0 & C_{m \phi} \\
\frac{C_{c u}}{\Delta t} & 0 & V_{c m} & K_{c c} & -K_{c \phi} \\
\frac{A_{\phi u}}{\Delta t} & 0 & S_{\phi m} & S_{\phi c} & K_{\phi \phi}
\end{array}\right\|^{n-1} \cdot\left|\begin{array}{c}
\hat{U}_{s} \\
\hat{p} \\
\hat{C}_{M} \\
\hat{C}_{c} \\
\hat{\phi}_{s}
\end{array}\right|^{n}= \\
& \left|\begin{array}{c}
\hat{F}_{u} \\
-\hat{Q}_{f / s} \cdot \Delta t \\
\hat{Q}_{m / s} \\
\hat{Q}_{c / s}+\hat{\Omega}_{c} \\
\hat{\Omega}_{s}
\end{array}\right|^{n}+\left|\begin{array}{c}
\hat{0} \\
-K_{u p}^{T} \cdot \hat{U}_{s} \\
\frac{C_{m m}}{\Delta t} \cdot \hat{C}_{M}+V_{m p} \cdot \hat{p}+C_{m \phi} \cdot \hat{\phi}_{s} \\
\frac{C_{c c}}{\Delta t} \cdot \hat{C}_{c}+\frac{C_{c u}}{\Delta t} \cdot \hat{U}_{s}+V_{c m} \cdot \hat{C}_{m}-K_{c \phi} \cdot \hat{\phi}_{s} \\
K_{\phi \phi} \cdot \hat{\phi}_{s}+S_{\phi m} \cdot \hat{C}_{M}+S_{\phi c} \cdot \hat{C}_{c}
\end{array}\right|
\end{aligned}
$$

The multiphasic periprosthetic healing tissue is implemented in Matlab(c) language. An axisymmetric 2D quad element with 8 nodes was developed. A quadratic interpolation functions was used for displacement $U_{s}$, growth factor concentration $C_{M}$, osteoblast concentration $C_{c}$ and solid fraction $\phi_{s}$. A linear interpolation field is used for the mean pressure $p$ into the porous media.

\section{Application}

In previous in vivo studies with an experimental cylindrical micro motion device (canine implant) representing the interface of a human implant (Vestermark et al., 2004), we have identified an association between the sites where peri-implant bone forms, and the experimentally determined mechanical and biological properties of the resulting interface. The distribution of new-formed tissue around the PMMA implant with no HA coating showed a polar symmetry $(r, \theta, z)$ with a variable level of mineralization in the radial direction $\overrightarrow{e_{r}}$. The meshing of the periprosthetic zone is plotted in Figure 3, this mesh is composed with 98 element in radial direction $\overrightarrow{e_{r}}$ and 1 element in axial direction $\overrightarrow{e_{z}}$. The meshing involved 591 nodes and 3546 DOF. It is refined at the implant surface $(I S)$ and at the drill hole surface $(D H S)$, the minimal thickness in radial direction at the drill hole surface was $1.10^{-2} \mathrm{~mm}$. In the zone between the implant surface $(I S)$ and the drill hole surface $(D H S)$, the element thickness was $2.10^{-2} \mathrm{~mm}$. The mesh was identical to that used in a preceeding study where a finite volume resolution was used (Ambard et al., 2006). We plotted in Figure 2 , the influence of the element number and time step $\Delta t$ on the convergence of the variation of the solid fraction $\phi_{s}$. We observed that a mesh of 100 elements provided very satisfying results. 


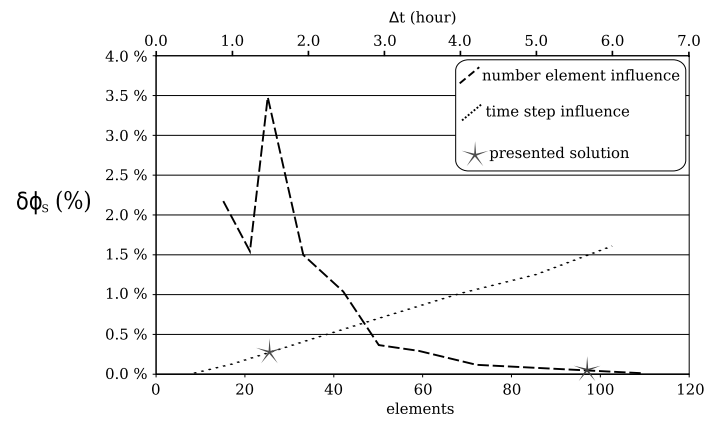

Figure 2. Influence of the mesh size and the time step on the solid fraction $\phi_{s}$ at 14 days

The time step was $\Delta t=1.5$ hour and the healing process was evaluated up to 14 days. The choice of time step was a compromise between physiological and biological reality and numerical constraints. The model requiered a sufficient time step to predict significant evolution in the reactive porous medium. It was also necessary to take into account the cyclic load of a daily activity. Finally, the time step was updated according to the solid fraction formation between $6 \%$ and $96 \%$. In fact, modifications of the selected time step showed a very low influence on the predicted mineralization. As shown in Figure 2, we found a maximal variation of $0.25 \%$ between 0.5 hour and 1.5 hour. The implant radius was: $3.25 \mathrm{~mm}$ (implant surface $I S$ ), the drill hole radius was: $4 \mathrm{~mm}$ (drill hole surface $D H S$ ); the investigated domain was $7 \mathrm{~mm}$ (host bone surface $H B S$ ), the thickness was $2 \mathrm{~mm}$.
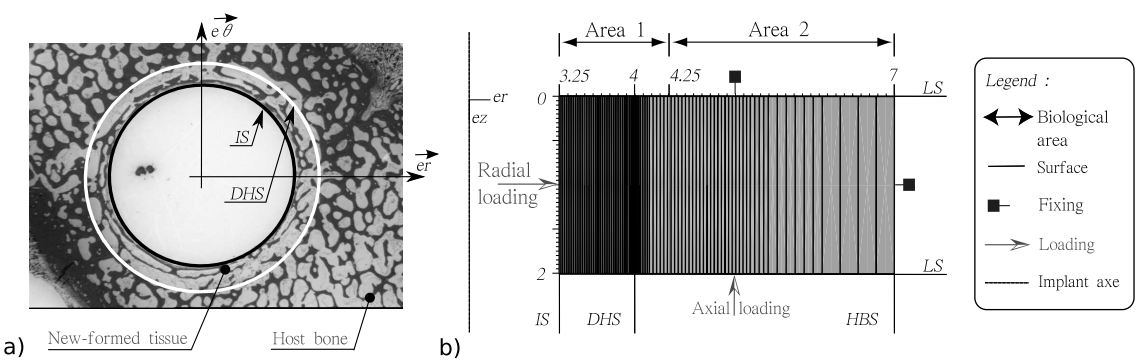

Figure 3. a) Histomorphometric data of the implant; b) Meshing and boundary conditions

During the healing process, the material properties were updated according to the amount of bone matrix $\phi_{s}$ as shown in Table 1. For the Young modulus [18] and 
Poisson ratio [19], we used the power functions. While for the permeability law, we used a Koseny-Carman's model function of Equations [20] and [21].

$$
\begin{aligned}
& E\left(\phi_{s}\right)=1220 \cdot \phi_{s}^{1,8891} \\
& v\left(\phi_{s}\right)=0,172 \cdot \phi_{s}^{0,27} \\
& \frac{k}{\mu}\left(\phi_{s}\right)=\frac{2,592 \cdot 10^{-5} \cdot \phi_{f}^{4,668}}{10^{5} \cdot S_{v}^{2}} \\
& S_{v}=323 . \phi_{f}-939 \cdot \phi_{f}^{2}+1340 . \phi_{f}^{3}-1010 . \phi_{f}^{4}+288 . \phi_{f}^{5}
\end{aligned}
$$

Table 1. Mechanical properties

\begin{tabular}{lll}
\hline Immature bone & Host bone & References \\
\hline $6 \leq \phi_{s} \leq 70 \%$ & $\phi_{s}=50 \%$ & \\
\hline $6 \leq E\left(\phi_{s}\right) \leq 600 M p a$ & $E=5000 \mathrm{Mpa}$ & (Lacroix, 2000; Fung, 1981) \\
\hline $0.1 \leq v\left(\phi_{s}\right) \leq 0.175$ & $v=0.27$ & (Lacroix, 2000; Fung, 1981) \\
\hline $11 \geq \frac{k}{\mu}\left(\phi_{s}\right) \geq 0.015 \mathrm{~mm}^{4} / N . s$ & $\frac{k}{\mu}=0.02 \mathrm{~mm}^{4} / N . s$ & (Arramon et al., 2001) \\
\hline
\end{tabular}

Table 2. Biological parameters of tissue

\begin{tabular}{llll}
\hline Physics & Parameters & Units & References \\
\hline Growth factor dispersion & $D_{M}=4,8.10^{-6}$ & $\mathrm{~mm}^{2} / \mathrm{s}$ & (Maheshwari et al., 1998) \\
\hline Osteoblast cells dispersion & $D_{c}=2,5.10^{-7}$ & $\mathrm{~mm}^{2} / \mathrm{s}$ & (Dee-Kay et al., 1999) \\
\hline Chemotaxis coefficient & $\chi_{c}=1.10^{-5}$ & $\mathrm{~mm}^{5} / \mathrm{s.ng}$ & (Maheshwari et al., 1998) \\
\hline Haptotactic coefficient & $h_{c}=1.10^{-5}$ & $\mathrm{~mm}^{5} / \mathrm{s}$ & (Friedl et al., 1998) \\
\hline Proliferation speed & $\alpha_{c}=1,9.10^{-10}$ & $\mathrm{~mm}^{3} / \mathrm{cell.s}$ & (Bailon-Plaza et al., 2001) \\
\hline Inhibition of the proliferation & $N_{c}=1.10^{3}$ & $\mathrm{cell} / \mathrm{mm}^{3}$ & (Bailon-Plaza et al., 2001) \\
\hline Synthesis speed & $\alpha_{s}=2.10^{-9}$ & $\mathrm{~mm}^{6} / \mathrm{cell.ng.s}$ & (Bailon-Plaza et al., 2001) \\
\hline
\end{tabular}

Biological parameters summarized in Table 2 allowed a satisfying correlation with homogeneous histomorphometric data to be obtained (Ambard et al., 2006). The fluorescence analysis (Vestermark et al., 2004) allowed the zone of main biological activity to be observed close from the implant (Area 1, Figure 3b). Initially, cells and growth factors were homogenously distributed: $C_{c}^{0}=1.10^{3} \mathrm{cell} / \mathrm{mm}^{3}$, $C_{M}^{0}=0.2 \mathrm{ng} / \mathrm{mm}^{3}$. As boundary conditions, the daily physical activities were modeled by imposed displacements $U_{r}(t)$ and $U_{z}(t)$ were sine functions of 24 hours period during 14 days, magnitude $\pm 0.2 \mathrm{~mm}$ which induced volume strain $\Delta v / v_{0}$ into the periprosthetic tissue: $-28 \%,-19 \%,-14 \%, 0 \%$, and $+8 \%$. To highlight the role of convective and diffusive transport for the classification of tissue healing, we adapted a dimensionless Péclet number such as $\left(P e=r_{d} \cdot\left\|\vec{q}_{f / s}\right\|_{\text {max }} / D_{M}\right) ; r_{d}$ being 
the radius of the drill hole $D H S$. The mechano-biological model predicted homogenous and heterogeneous periprosthetic tissue healing. When volume strain evolved from compression $\left(\Delta v / v_{0} \geq 0\right)$ to traction $\left(\Delta v / v_{0} \leq 0\right)$, the bone fraction $\phi_{s}$ at the implant surface (IS) was decreasing from $53 \%$ to $25 \%$ (Figure $4 a$ ), the osteoblast concentration $C_{c}$ and the growth factor concentration $C_{M}$ (Figure $4 \mathrm{~b}$ and c) followed the same progression. At the drill hole $(D H S)$, growth factors and cells concentrations decreased specially for compression (Figure $4 \mathrm{~b}$ and $\mathrm{c}$ ). The bone fraction showed a progression $(50 \%-60 \%)$ opposed to that observed on the implant. As shown in Figure 4d, the Péclet number increased from 2 to 7 and it was nil when no strain was imposed into the tissue $\left(\Delta v / v_{0}=0\right.$, no forced convection). For magnitude greater than 3 obtained for compression, it appeared that the mechano-biological couplings (combination of convective and diffusive transport) played a significant role on the periprosthetic healing.

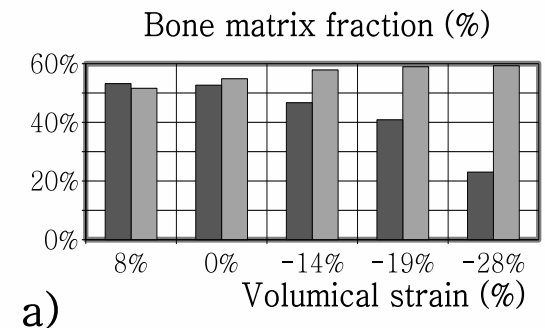

a)

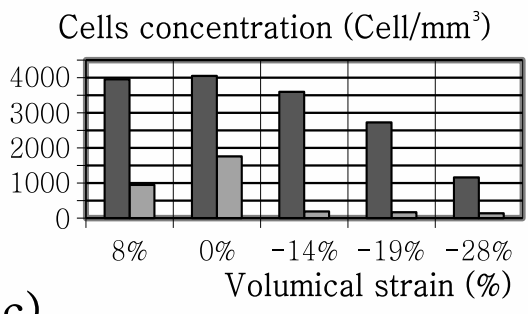

c)

Implant surface (IS)
Growth factor $\left(\mathrm{ng} / \mathrm{mm}^{3}\right)$
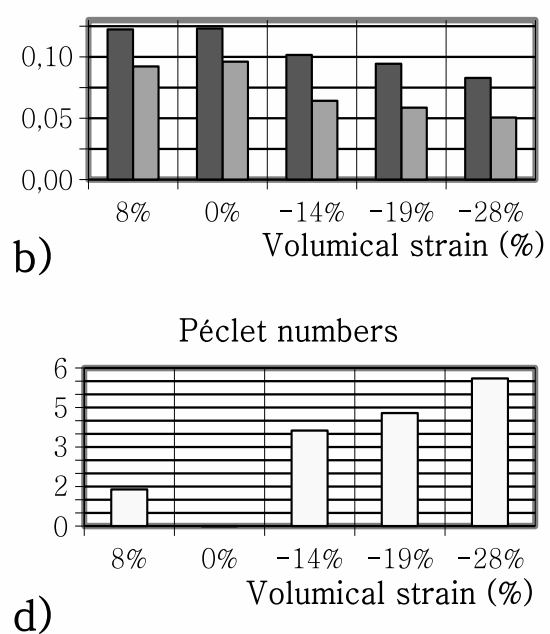

$\square$ Drill hole surface (DHS)

Figure 4. Predicted healing at 14 days: (a) bone fraction $\phi_{s}$; (b) growth factor concentration $C_{M}$; (c) osteoblast concentration $C_{c}$; (d) Péclet number

Finally, the sensitivity analysis using volume strain as variable showed that compression $\left(\Delta v / v_{0} \geq 0\right)$ was unfavourable to homogenous distribution of new-formed bone into the gap surrounding the implant. This could contribute to the formation of sclerotic bone rim observed in vivo in our preliminary studies in canine implant (Mouzin et al., 2001; Vestermark et al., 2004). 


\section{Conclusion}

We proposed an original multiphasic model that associated poroelasticity, reactive media, and biomathematical equations. We implemented a specific finite element resolution involving mechanical and biological parameters as output measures.

Comparison with previous studies in an experimental animal model showed promising results to conclude that our central hypothesis coupling cell migration, growth factor diffusion and deformable porous media was valid at the first order. The numerical model allowed heterogeneous bone formation to be predicted which is quite innovative in the domain of implant fixation. Favour the homogenous healing controlling growth factor concentration and sources (bioactive coating, bone graft, protein) and evaluate the clinical procedure specially in implant revision (press-fit, gap size, cell concentration, residual solid fraction) could be a relevant strategy.

Despite our encouraging results, it seems that the robustness of future numerical predictions will find, at least, two limitations. First, the coupling of governing equations in biology to poroelasticity increased the number of uncontrolled in vivo parameters such as the evolving permeability, the delivering properties of growth factors, the diffusive and proliferation properties of cells. These points will have to be investigated. Second, we observed the significant role of the mechanical signal on tissue formation, whereas the in vivo mechanical loading in time and magnitude, is poorly known. This would be a pertinent orientation to improve the model reliability.

Acknowledgements

JE Bechtold PhD (MORF Minneapolis MN, USA); Pr. K. Søballe (Aarhus University Hospital, Denmark).

\section{References}

Alliston T. N., Derynck R., Skeletal growth factors., Lippincott Williams /\& Wilkins, chapter Transforming Growth Factor- $\beta$ in Skeletal Development and Maintenance, p. 233-249, 2000.

Ambard D., Swider P., “ A predictive mechano-biological model of the bone-implant healing”, European Journal of Mechanics - A/Solids, vol. 25, n 6, p. 927-937, November-December, 2006.

Ames-William F., Numerical methods for partial differential equations, Academic press New York San Fransisco, 1977.

Arramon Y., Naumann E., Bone Mechanics Handbook, chapter The intrinsic Permeability of Cancellous Bone, p. 25-17, 2001.

Bailon-Plaza A., Van-Der-Meulen M., “ A Mathematical Framework to Study the Effects of Growth Factor Influences on Fracture Healing”, Journal of theoritical Biologie, vol. 212, p. 191-209, 2001. 
Bechtold J. E., Kubic V., Søballe K., “ A controlled experimental model of revision implants: Part I. Development”, Acta Orthop Scand, vol. 72, n 6, p. 642-649, Dec, 2001a.

Bechtold J. E., Mouzin O., Kidder L., Søballe K., “ A controlled experimental model of revision implants: Part II. Implementation with loaded titanium implants and bone graft”, Acta Orthop Scand, vol. 72, n 6, p. 650-656, Dec, 2001 b.

Carter D. R., "Mechanical loading history and skeletal biology”, Journal of Biomechanics, vol. 20, p. 1095-1109, 1987.

Carter D. R., Blenman P. R., al, “ Correlations between mechanical stress history and tissue differentiation in initial fracture healing”, J. Orthop. Res, vol. 6, p. 736-748, 1988.

Conover C. A., Skeletal Growth factors, Philadelphia, Lippincott Williams /\& Wilkins, chapter Insulin-like Growth Factors and the Skeleton, p. 101-116, 2000.

Dee-Kay C., Thomas T., X, “ Osteoblast population migration characteristics on substrates modified with immobilized adhesive peptides”, Biomaterials, vol. 20, p. 221-227, 1999.

Dhatt G., Touzot G., Une présentation de la méthode des éléments finis. Deuxième Edition, Université de Compiegne, 1984.

Friedl P., Zanker K. S., Brocker E. B., “ Cell migration strategies in 3-D extracellular matrix: differences in morphology, cell matrix interactions, and integrin function", Microsc Res Tech, vol. 43, n 5, p. 369-378, Dec, 1998.

Fung Y., Biomechanics Mechanical properties of living tissues, New York, Springer Verlag, 1981.

Hahn M., Vogel M., X, “ Bone structure changes in hip joint endoprosthesis implantation over the course of many years. A quantitative study”, Chirurg, vol. 59, p. 782-787, 1988.

Huiskes R., Ruimerman R., van Lenthe G. H., Janssen J. D., “ Effects of mechanical forces on maintenance and adaptation of form in trabecular bone", Nature, vol. 405, n 6787, p. 704706, Jun, 2000.

Kibbin M. B., “ The biology of fracture healing in long bones”, J Bone Joint Surg.Am, vol. 79, p. 1938-1941, 1997.

Kunzler T. P., Drobek T., Schuler M., Spencer N. D., " Systematic study of osteoblast and fibroblast response to roughness by means of surface-morphology gradients", Biomaterials, vol. 28, p. 2175-2182, 2007.

Lacroix D., Simulation of tissue differentiation during fracture healing, $\mathrm{PhD}$ thesis, University of Dublin, 2000.

Linckart T. A., Mohan S., "Growth factors for bone growth and repair”, Bone, vol. 19, p. 1-12, 1996.

Maheshwari G., Lauffenburger D. A., “ Deconstructing (and reconstructing) Cell Migration”, Microscopy research and technique, vol. 43, p. 358-368, 1998.

Meinel L., Zoidis E., Zapf J., Hassa P., Hottiger M. O., Auer J. A., Schneider R., Gander B., Luginbuehl V., Bettschart-Wolfisberger R., Illi O. E., Merkle H. P., von Rechenberg B., "Localized insulin-like growth factor I delivery to enhance new bone formation", Bone, vol. 33, n 4, p. 660-672, Oct, 2003.

Mouzin O., Søballe K., Bechtold J. E., “ Loading improves anchorage of hydroxyapatite implants more than titanium implants”, J Biomed Mater Res, vol. 58, n 1, p. 61-68, 2001. 
Prendergast P. J., Huiskes R., Søballe K., “ ESB Research Award 1996. Biophysical stimuli on cells during tissue differentiation at implant interfaces", J Biomech, vol. 30, n 6, p. 539-548, Jun, 1997.

Puleo D. A., et al L. A. H., “ Osteoblast responses to orthopedic implant materials in vitro”, $J$ Biomed.Mater.Res, vol. 25, p. 711-723, 1991.

Søballe K., Hansen E. S., al, " Hydroxyapatite coating converts fibrous tissue to bone around loaded implants”, J Bone Joint Surg, vol. 75, p. 270-278, 1993.

Søballe K., Jensen T. B., Mouzin O., Kidder L., Bechtold J. E., “ Differential effect of a bone morphogenetic protein-7 (OP-1) on primary and revision loaded, stable implants with allograft”, J Biomed Mater Res A, vol. 71, n 4, p. 569-576, Dec, 2004.

Van-Der-Meulen M. C. H., Huiskes R., “ Why mechanobiology ”, Journal of Biomechanics, vol. 35, p. 401-414, 2002.

Vestermark M. T., Bechtold J. E., Swider P., Søballe K., “ Mechanical interface conditions affect morphology and cellular activity of sclerotic bone rims forming around experimental loaded implants”, J Orthop Res, vol. 22, n 3, p. 647-652, May, 2004.

Zaman M. H., Kamm R. D., Matsudaira P., Lauffenburger D. A., “ Computational Model for Cell Migration in Three-Dimensional Matrices", Biophysical Journal, vol. 89, p. 13891397, 2005. 